\title{
MEASUREMENT OF THE IMPUSLIVE FORCE GENERATED BY COLAPSING BUBBLE CLOSE TO A SOLID BOUNDARY
}

\author{
Milos MULLER, Jiri UNGER, Jakub HOLATA, Patrik ZIMA •
}

\begin{abstract}
The article presents experimental results of the acoustical and optical study of cavitation bubble collapse close to a solid boundary in water. The bubble was generated by discharge of low-voltage capacitor into a couple of wires closing a simple circuit. Different distances from the solid wall and different maximum bubble radii were studied. The bubble radius was studied using time-resolved photography and by PVDF film sensor glued on the solid boundary. The illumination was provided by high-power led diode. Synchronization of the system was provided by pulse generator connected to an oscilloscope. The impact power of the bubble to the wall was estimated from the time-resolved photography of the bubble and from the PVDF film sensor signal. The PVDF film sensor calibration was performed by a pendulum test to estimate the impact force.
\end{abstract}

\section{INTRODUCTION}

Understanding the collapse of a single cavitation bubble is essential to a number of fields including medical, marine or pump industries. Several techniques are commonly used for laboratory production of a single bubble. These include laser induction [1-2], spark generation [3-6] and acoustic generation [7]. The investigation of laser-induced bubbles and the accompanying shock waves was invoked by the massive utilization of lasers in medical applications [2]. The spark-generated bubbles are often used to study underwater explosions [3]. Typically, they are produced by high voltage, however, one can use low voltage [6] providing that the electrodes are in direct contact. The authors' intention is to study bubbles for hydrodynamic applications, namely collapse of hydrodynamic cavitation bubble at different distances from the wall including the zero distance from the wall. For this purpose, an experimental setup utilizing generation of bubbles in a heated channel is being developed. Such method is not new; see for example [8], however, it requires another source to initialize the bubble collapse. Therefore, for the purpose of this early study, a proven setup with spark-generated bubbles will be used.

\footnotetext{
- Miloš Muller, Faculty of Mechanical Engineering, Technical University of Liberec, Studentská 2, 46117, Liberec 1, Czech Republic, milos.muller@tul.cz

Jiri Unger, Faculty of Mechanical Engineering, Technical University of Liberec, Studentská 2, 46117, Liberec 1, Czech Republic, jiri.unger@tul.cz

Jakub Holata, Faculty of Mechanical Engineering, Technical University of Liberec, Studentská 2, 46117, Liberec 1, Czech Republic, j.holata@seznam.cz

Patrik Zima, Department of Thermodynamics, Institute of Thermomechanics, v.v.i., Academy of

Sciences of the Czech Republic, Dolejškova 5, 18200 Praha, Czech Republic, zimap@it.cas.cz
}

This is an Open Access article distributed under the terms of the Creative Commons Attribution License 2.0, which permits unrestricted use, distribution, and reproduction in any medium, provided the original work is properly cited. 
It is known from the theory [9-10] that the bubble oscillations are strongly influenced by the presence of the wall. The bubble is attracted to the wall during the collapse causing damage to the wall material. The damage is caused by several well-known effects: rapid re-entrant liquid micro jet, strong shock wave, the so-called splashing effect or combined action of these and other (for example electrochemical) effects.

\section{EXPERIMENTAL SETUP}

Figure 1 shows the experimental setup for investigation of a single spark-generated cavitation bubble. The bubble is created between two copper electrodes placed in a glass bath filled with tap water. The electrodes are in direct contact to enable the use of low voltage. The spark is produced by two parallel capacitors (4700 $\mu \mathrm{F})$, which are discharged through a relay. The temporal evolution of the bubble radius is recorded by the high-speed Redlake Motion Pro CCD camera with 10000 fps for all experiments. The illumination of the experiment is provided by the power led diode. To record the pressure response during the bubble collapse a PVDF film sensor DT1-028K W/TH (Measurement Specialties) is used. The signals are digitalized using the oscilloscopic card with the sampling frequency $60 \mathrm{MHz}$.

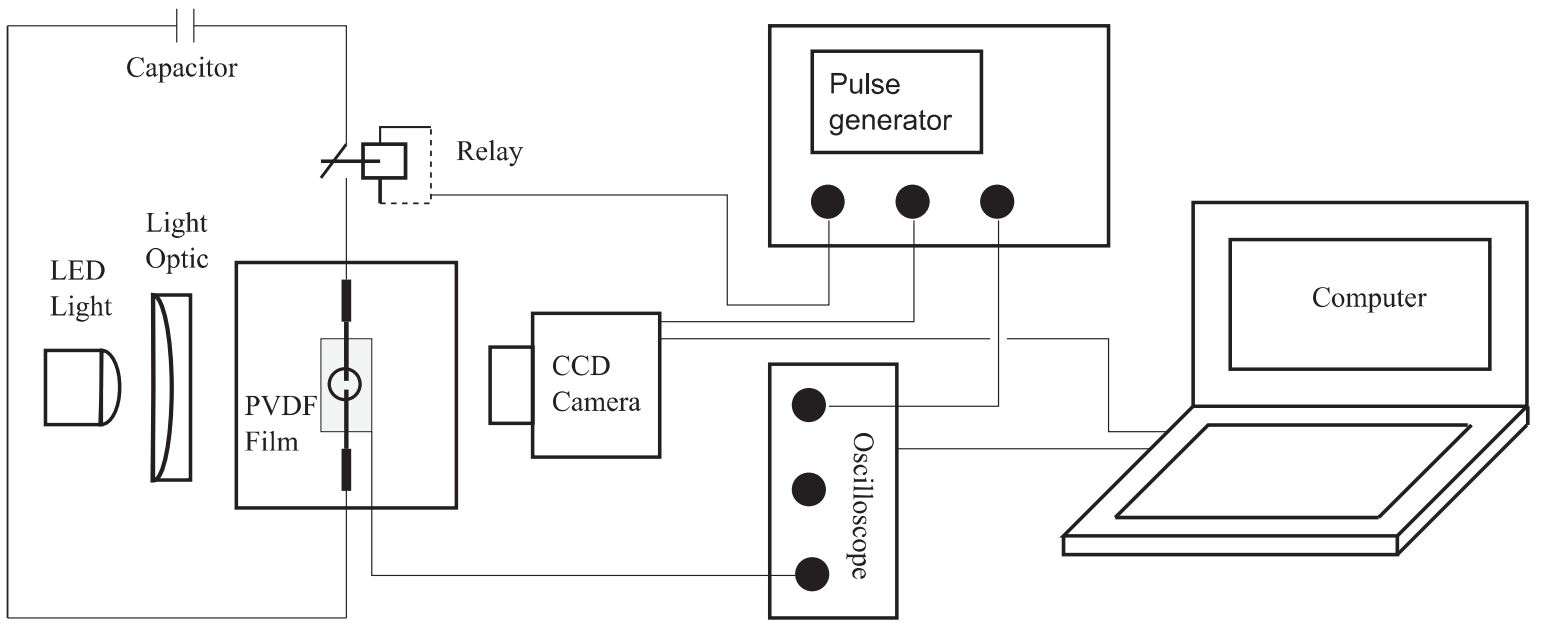

Figure 1: Experimental setup

The calibration of the PVDF film sensor is provided using the pendulum. The schematic illustration of the calibration test is shown in Figure 2 (right). The impact force of the pendulum can be estimated from the following equation

$$
F_{i}={ }_{\tau}^{m}\left(v_{1}+v_{2}\right),
$$

where $m$ is the mass of the sphere, $\tau$ is the impact duration time and $v_{1}$ and $v_{2}$ are the velocities just before and after impact, respectively, which can be calculated from the starting angle and the angle after impact as

$$
v_{1,2}=\sqrt{2 g h_{1,2}} .
$$



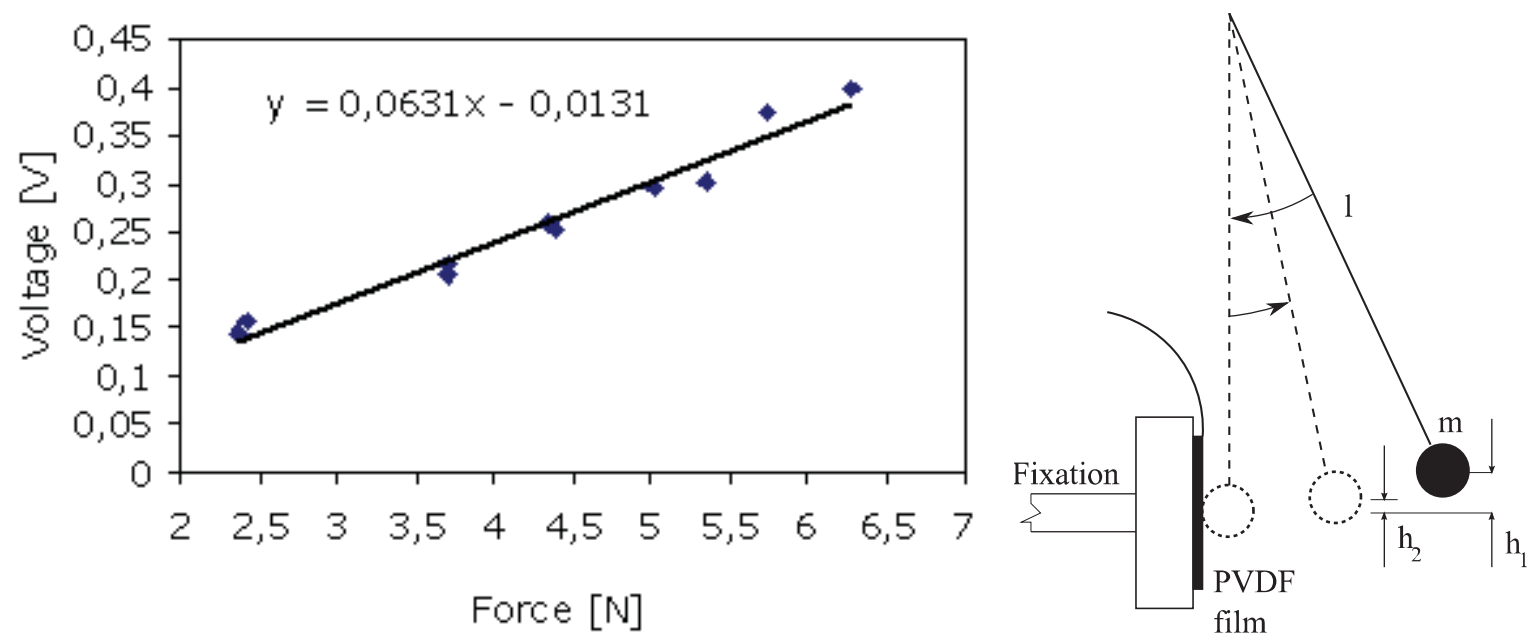

Figure 2: PVDF film sensor calibration setup (right) and calibration curve (left)

The results obtained during the calibration procedure are shown in Figure 2 (left). The plotted data represent RMS values of the voltage signal with duration $\tau$. The approximation curve allows to extend the calibration data to regions of higher impact intensities.

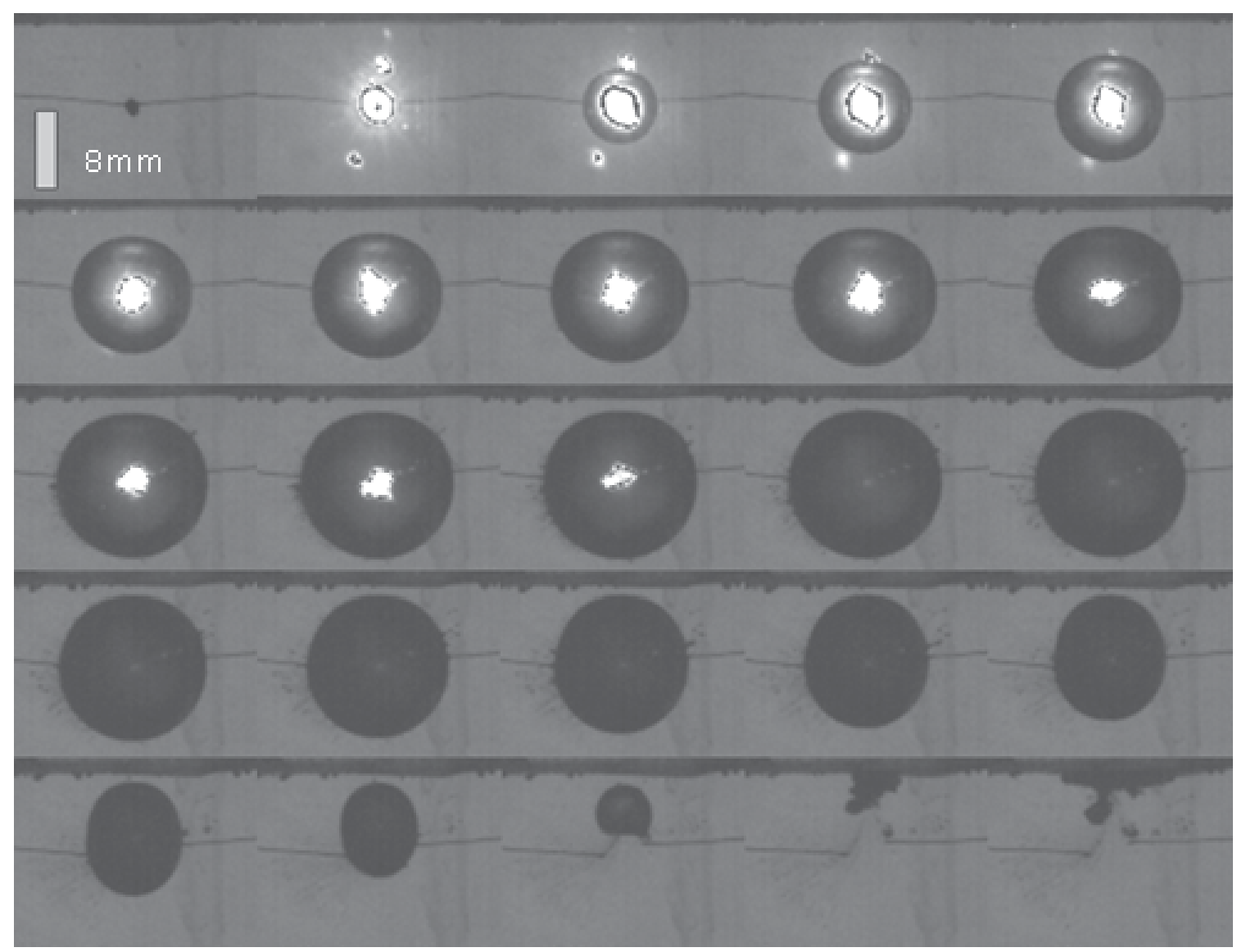

Figure 3: Cavitation bubble dynamics close to a solid boundary for the bubble-wall distance $8 \mathrm{~mm}$ (frame interval $100 \mu \mathrm{s}$ ). The figures show the first expansion and the first collapse. 


\section{Results}

A typical sequence of a bubble collapsing close to a solid boundary from our experiments is shown in Figure 3 and the corresponding PVDF film sensor signal in Figure 4. The bubble expansion starts at time 4.3 milliseconds, which corresponds to the delay time of the relay. The burst on the second figure is caused by the camera overexposure. After the plasma creation the bubble starts to grow. The first collapse produces a strong impact against the solid wall, which is visible from PVDF film sensor signal in Figure 4. The impact of the bubble jet on the surface is indicated by a small peak between the first and the second collapse. The second collapse starts when the bubble reaches the wall. The strength of the first and following collapses is strongly influenced by the distance of the bubble from the wall and the maximum bubble size, which could be expressed by a ratio

$$
\gamma=\frac{h}{R_{\max }}
$$

where $R_{\max }$ is the maximum bubble radius and $h$ the initial bubble distance from the wall. The studied range of $\gamma$ in this article was $0,84-2,9$. For higher values of $\gamma$ the bubble wall interaction is provided by the shock wave. For $\gamma>1$ and $\gamma<3$ the bubble initially collapses in its original position. However, during the rebound the bubble moves towards the wall and the second collapse occurs on the wall. For $\gamma<1$ the bubble wall first interacts with the solid wall during the first expansion phase. All the collapses in this case occur on the wall.

The forces generated during the bubble collapses are shown the in Figure 5 for the first collapse and in Figure 6 for the second collapse. It can be seen that the first bubble collapse produces higher impact force value than the second collapse at the positions far from the wall (stand-off distance $16 \mathrm{~mm}$ ). This corresponds to the bubble behavior not influenced by the wall. The force of the first collapse is much lower than for the second collapse for the stand-off distance $13 \mathrm{~mm}$. This is probably due to the fact that the secondary collapse occurs directly on the wall.

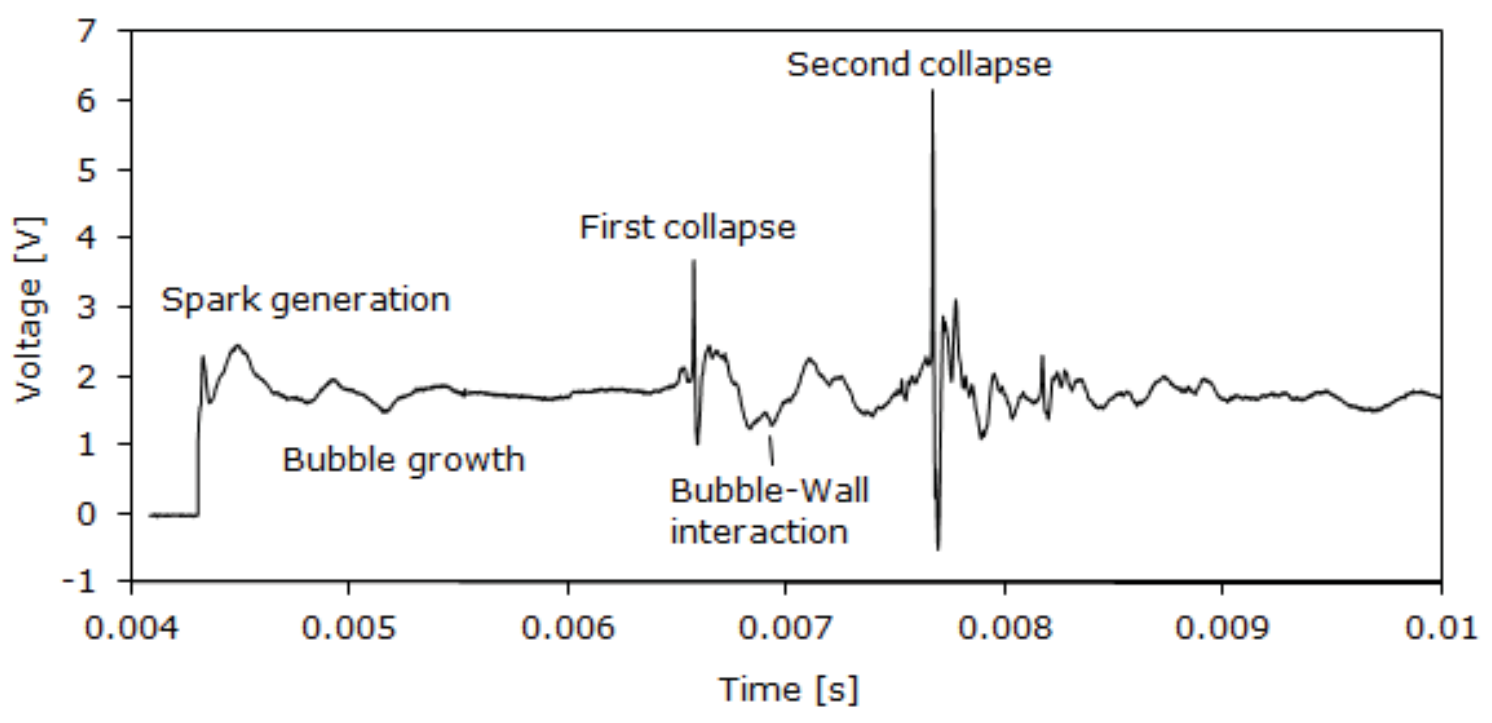

Figure 4: PVDF film sensor signal corresponding to the sequence from Figure 3. 


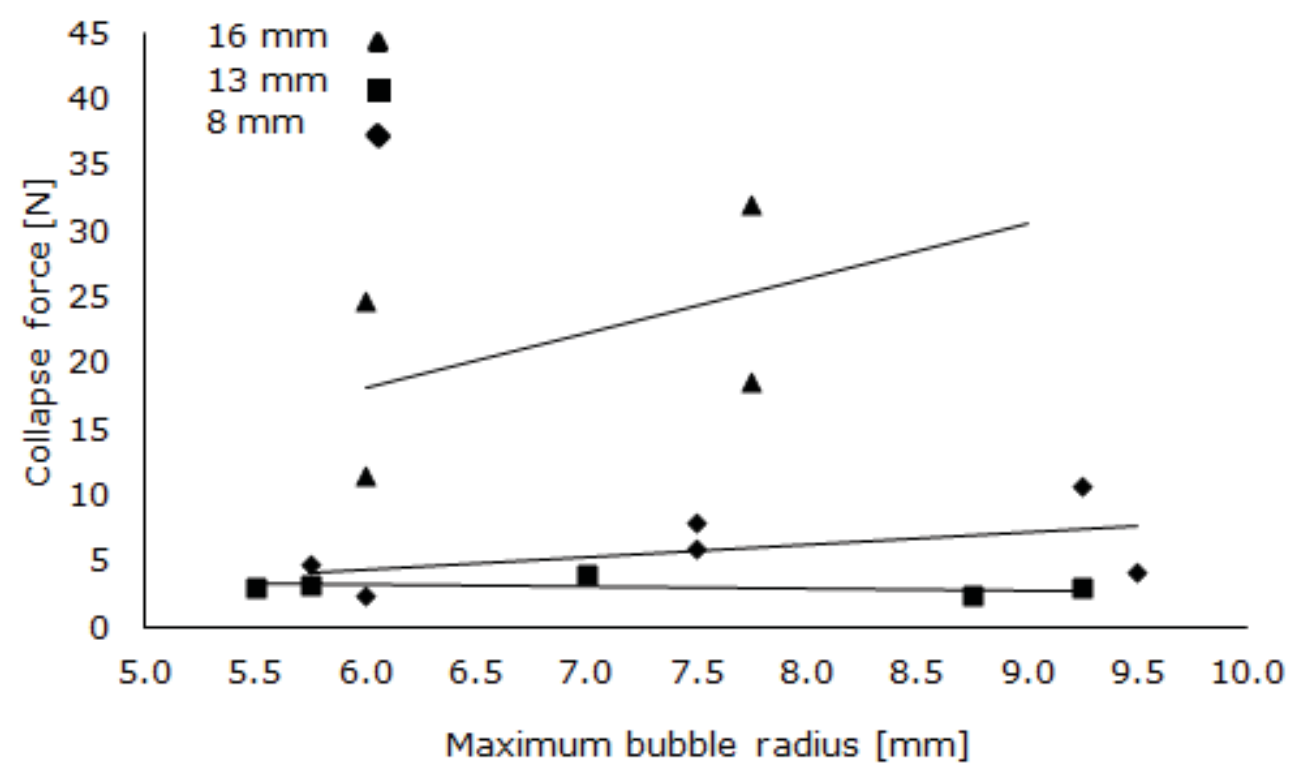

Figure 5: Impact force of the first bubble collapse. The bubble wall distances are 16,13 , and $8 \mathrm{~mm}$.

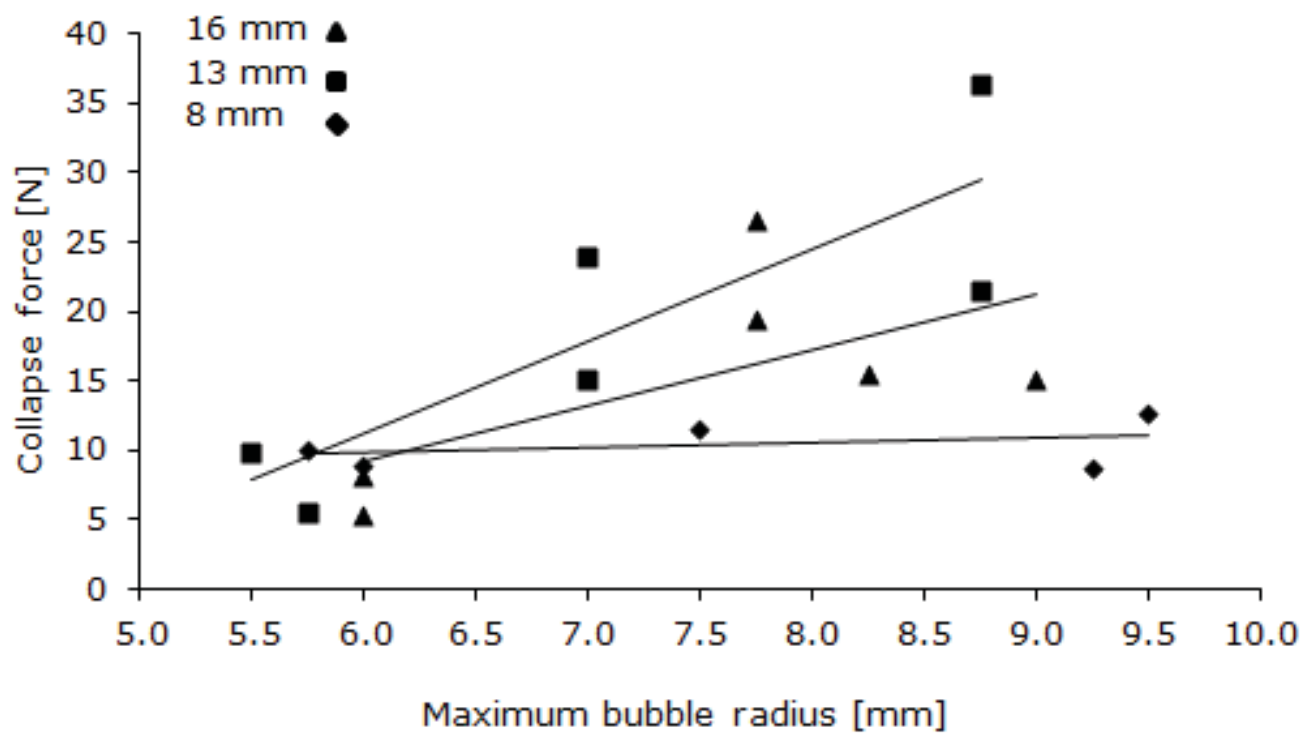

Figure 6: Impact force of the second bubble collapse. The bubble wall distances are 16,13 , and $8 \mathrm{~mm}$.

For bubble collapses close to the wall $(\gamma<1)$ the impact forces of the first collapse is slightly lower than the impact forces of the second collapse. This is probably due to the fact that the primary collapse starts in the breakdown position, but the secondary collapse starts directly on the wall.

\section{Conclusion}

The bubble collapse impact on the wall was studied using PVDF film sensor. The resulting impact forces depend on the stand-off distance and the interaction of the bubble with the wall. For bubbles far from the wall, the impact force by the first collapse is higher than the impact force of the second collapse. Bubbles collapsing directly on the wall can 
produce very high impact forces also during the second collapse. However, for medium stand-off distance the ratio between the impact forces produced during the first and second collapses changes very strongly as reported also in [5].

The method utilizing the PVDF film sensor as the solid wall seems to be adequate for investigation of the bubble collapse impact. However, it is necessary to consult the PVDF sensor record with the CCD camera record to identify the processes causing the impact.

\section{ACKNOWLEDGEMENT}

The authors wish to thank the Czech Science Foundation for providing financial support for grant no. 101/10/1428 and grant no. MSM 4674788501 of the Technical University of Liberec.

\section{REFERENCES}

[1] Lauterborn, W. and Bolle, H.: Experimental investigations of cavitation-bubble collapse in the neighborhood of a solid boundary, J. Fluid Mech., 72, 1975, 391399

[2] Vogel, A., Lauterborn, W., Timm, R.: Optical and acoustic investigations of the dynamics of laser-produced cavitation bubbles near a solid boundary, J Fluid Mech., 206, 1989, 299-338

[3] Chahine G. L.: Spark Generated Bubbles as Laboratory-Scale Models of Underwater Explosions and their use for Validation of Simulation Tools, Proceedings of the 66th Shock and Vibration Symposium,1995, Biloxi, MS

[4] Buogo, S., Plocek, J., Vokurka, K.: Efficiency of Energy Conversion in Underwater Spark Discharges and Associated Bubble Oscillations: Experimental Results, Acta Acustica united with Acustica, 95, 2009, 46-59

[5] Wang, Y. C., Chen, Y. W.: Application of piezoelectric PVDF film to the measurement of impulsive forces generated by cavitation bubble collapse near a solid boundary, Exp. Thermal Fluid Sci., 322, 2007, 403-414

[6] Lew, K. S. F., Klaseboer E., Khoo, B. C.: A collapsing bubble-induced micropump: an experimental study," Sensors Actuat. A, 133, 2007, 161-172

[7] Lauterborn, W., Kurz, T., Geisler, R., Schanz, D., Lindau, O.: Acoustic cavitation, bubble dynamics and sonoluminescence," Ultrasonics Sonochemistry, $14,2007,484-491$

[8] Helden, W., Geld, C., Boot, P.: Forces on bubbles growing and detaching in flow along a vertical wall, Int. J. Heat Mass Transfer, 38, 1995, 2075-2088

[9] Brennen Ch. E.: Cavitation and Bubble Dynamics, 1995, Oxford University Press

[10] Franc, J. P. and Michel J. M.: Fundamentals of Cavitation, 2004, Kluwer Academic Publisher 